This document is the accepted manuscript version of the following article:

Racles, C., Bele, A., Dascalu, M., Musteata, V. E., Varganici, C. D., Ionita, D., ... Opris, D. M. (2015). Polar-nonpolar

interconnected elastic networks with increased permittivity and high breakdown fields for dielectric elastomer transducers.

RSC Advances, 5(72), 58428-58438. http://doi.org/10.1039/C5RA06865G

\title{
ARTICLE
}

\section{Polar-nonpolar interconnected elastic networks with increased permittivity and high breakdown fields for dielectric elastomer transducers}

\author{
C. Racles ${ }^{a} *$, A. Bele ${ }^{a}$, M. Dascalu ${ }^{a}$, V. E. Musteata ${ }^{a}$, C. D. Varganici ${ }^{a}$, D. Ionita ${ }^{a}$, S. Vlad ${ }^{a}$, M. \\ Cazacu $^{a}$, S. J. Dünki ${ }^{b}$ and D. M. Opris ${ }^{\text {b* }}$
}

\begin{abstract}
Elastic materials with increased permittivity $\left(\varepsilon^{\prime}\right)$ were obtained in a three-step process starting from a hydroxyl endfunctionalized polydimethylsiloxane (PDMS) of a high molecular weight $\left(M_{w}=139 \mathrm{kDa}\right)$, trimethylsilyl end-blocked silicones that carry hydrosilane, cyanopropyl and hexyl groups $\mathbf{P}_{\mathbf{x}}$ (where $\mathrm{x}$ represents the mol\% of cyanopropyl groups), and tetraethoxysilane (TEOS). The hydrosilane groups of $\mathbf{P}_{\mathbf{x}}$ were first hydrolyzed and the formed hydroxyl groups were subsequently reacted with partially hydrolyzed TEOS and further used as high $\varepsilon^{\prime}$ components, cross-linkers, and reinforcing agents for the PDMS matrix. A high wt\% of polar component $\mathbf{P}_{\mathrm{x}}$ was incorporated into the nonpolar PDMS matrix by forming interconnected networks. Thermal (DSC, DMA) and morphological investigations (SEM) show the biphasic morphology of the networks. The dielectric, mechanical, and electromechanical properties of the films were investigated. Materials with good elastic properties, increased $\varepsilon^{\prime}$, and high breakdown field $\left(E_{b}\right)$ were obtained. The best material has an elastic modulus of $800 \mathrm{kPa}$ at $10 \%$ strain, an $\varepsilon^{\prime}=4.5$, and a maximum actuation strain of $8 \%$ at $E_{b}=56 \mathrm{~V} / \mu \mathrm{m}$.
\end{abstract}

\section{Introduction}

Dielectric elastomer transducers (DET) are devices able to convert electrical energy to mechanical energy or vice versa [1]. DET are finding applications in robotics [2-6] artificial muscles [7,8] medical devices [9-13], replacements of space engines $[14,15]$ or in energy harvesting from different sources like wind [16], human motion [17, 18], or waves [19-21]. As dielectric, several elastomers such as silicones, polynitrile, polyacrylate, and natural rubbers as well as composites thereof have been used [22, 23].

The transducer performance is directly influenced by the stiffness and the permittivity $\left(\varepsilon^{\prime}\right)$ of the dielectric. Large actuation strains are expected for materials that have a high $\varepsilon^{\prime}$ and a low elastic modulus. However, in terms of stress, a low elastic modulus $(Y)$ value is not always of advantage, since the maximum stress attainable in an actuator increases for stiff materials [21].

Silicones are characterized by a high flexibility of the Si-O bond $[24,25]$, which is responsible for the large achievable strains. Additionally, they are biocompatible, resistant to weathering, and have stable properties over a wide range of temperatures and frequencies $[26,27]$. Furthermore, silicones have good dielectric strength typically over $100 \mathrm{~V} / \mu \mathrm{m}$ and maintain their specific energy over a wide frequency domain [28]. Despite of these great

\footnotetext{
a. Petru Poni Institute of Macromolecular Chemistry, Aleea Grigore Ghica Voda 41A, lasi, 700487, Romania, e-mail: raclesc@icmpp.ro.

b. Empa, Swiss Federal Laboratories for Materials Science and Technology, Laboratory for Functional Polymers, Ueberlandstr. 129, CH-8600, Dübendorf, Switzerland, e-mail: Dorina.Opris@empa.ch.

+ Electronic Supplementary Information (ESI) available: $\left[{ }^{29} \mathrm{Si}\right.$ and ${ }^{1} \mathrm{H}$ NMR spectra, IR spectra, images of the films, SEM image of a blend with $\mathrm{P}_{x}$ particles, DSC curves, DMA, fatigue resistance tests, calculated actuation, mechanical and electromechanical properties of the optimized materials]. See DOI: 10.1039/x0xx00000x
}

properties, silicones have a low $\varepsilon^{\prime}$ of less than 3 .

The application potential of dielectric silicone materials would increase significantly if $\varepsilon^{\prime}$ could be increased while keeping the breakdown field strength and the elastic moduli at reasonable values. Attempts to increase the $\varepsilon^{\prime}$ of silicones include blending it with polarizable particles or with a polar component, chemical modification with polar side groups, or using a polar cross-linker [29-32].

When polar cross-linkers are used, homogenous silicone elastomers form, but unfortunately the increase in the $\varepsilon^{\prime}$ is low due to the limited amount of polar groups that can be incorporated [32].

A larger degree of functionalization of silicones can be achieved when the Si-O repeating units comprise a polar group [33, 34]. Cyanopropyl-modified silicones were recently used as fillers in a silicone elastomer matrix [31] cross-linked by hydrosilylation. Composites with polar filler content up to $39 \mathrm{wt} \%$, that reached $\varepsilon^{\prime}$ values as high as 7 were reported. However, the breakdown field $\left(E_{b}\right)$ decreased significantly with increasing the $\varepsilon^{\prime}$. Blending allows formation of materials with a polar phase physically dispersed into a nonpolar matrix. However, when the filler is not chemically bonded to the matrix, it can leak or can be extracted from the film, which leads to deterioration of the dielectric as well as mechanical properties in time.

We previously described the synthesis of nitrile containing silicone materials by cross-linking of copolymers containing different mol\% of cyanopropyl groups and hydroxyl end-groups [34]. Materials with $\varepsilon^{\prime}$ as high as 6.5 but rather poor elastic properties formed. To improve on this, the modified copolymers were used as high $\varepsilon^{\prime}$ components in a PDMS matrix with suitable mechanical properties. To avoid phase separation, a fast cross- 
linking reaction was used [34]. This simple method allowed crosslinking the homogenous solution and macro-phase separation was avoided. Unfortunately, the amount of polar material incorporated into the PDMS matrix was rather low and therefore the maximum achieved permittivity was only $\varepsilon^{\prime}=3.5$. Recently, a series of polymers containing various amounts of nitrile groups was reported and the dielectric properties of the formed polymers were investigated. The hydrosilylation was incomplete and some residual $\mathrm{Si}-\mathrm{H}$ groups were still present [35]. Although disappointing, these results suggest that it might be possible to use the unreacted $\mathrm{Si}-\mathrm{H}$ groups in subsequent reactions.

Thus, it was the aim of the present work to develop a synthetic strategy to elastomers that incorporate large amounts of polar cyanopropyl modified silicones by chemically bonding them to a nonpolar PDMS matrix. For this, trimethylsilyl end-blocked polysiloxanes $\mathbf{P}_{\mathbf{x}}$ containing different proportions of cyanopropy ( $\mathrm{x}$ mol\%), hexyl and $\mathrm{Si}-\mathrm{H}$ groups were used as polar components [35]. The unreacted $\mathrm{Si}-\mathrm{H}$ groups of $\mathbf{P}_{\mathbf{x}}$ were used to interconnect $\mathbf{P}_{\mathbf{x}}$ with the hydroxyl end-groups of PDMS, in a three-step process, which involves hydrolysis and condensation reactions, leading to polarnonpolar interconnected networks. These reactions were carefully followed step by step by FT-IR. The thermal, dielectric and mechanical properties of the obtained films, as well as their morphology, were investigated by a combination of techniques: DSC, DMA, impedance spectroscopy, tensile measurements, fatigue tests, SEM. Their actuation performance was tested in circular membrane actuators.

\section{Experimental}

\section{Materials and Methods}

The following reagents were used as received: a linear hydroxyl end-functionalized PDMS $\left(M_{w}=139 \mathrm{kDa}\right)$ from $A B C R$, tetraethyoxysilane (TEOS) from Fluka, and dibuthyltin dilaurate (DBTDL) from Sigma-Aldrich. Tris(hydroxymethylaminomethane)end-functionalized PDMS oligomer with 19 dimethylsiloxane units was prepared following our previously reported method [36] and used as non-aqueous surfactant. All solvents were reagent grade and used as received.

The infrared spectra were recorded on a Bruker Vertex 70 FT-IR instrument, in transmission mode, in the $300-4000 \mathrm{~cm}^{-1}$ range (resolution $2 \mathrm{~cm}^{-1}, 32$ scans), at ambient temperature. Differential scanning calorimetry (DSC) investigations were done on a Pyris Diamond DSC (Perkin Elmer USA) instrument. NMR measurements were conducted on a Bruker Avance III 400 NMR spectrometer using a $5 \mathrm{~mm}$ BBO Prodigy ${ }^{\mathrm{TM}}$ CryoProbe at 400.18, 100.63 and 79.50 $\mathrm{MHz}$, respectively. For SEM investigation, the films were fractured in liquid nitrogen and the cross-section surface was examined with an Environmental Scanning Electron Microscope (ESEM) type Quanta 200, operating at $30 \mathrm{kV}$ with secondary electrons. Dynamic mechanical analysis (DMA) measurements were conducted on a PerkinElmer Diamond DMA instrument, in tension mode, under nitrogen atmosphere. Temperature scans were performed on films with dimension of $9 \times 9 \times 0.4 \mathrm{~mm}^{3}$ at a heating rate of $2{ }^{\circ} \mathrm{C} / \mathrm{min}$ from $150{ }^{\circ} \mathrm{C}$ to $200^{\circ} \mathrm{C}$ and at $1 \mathrm{~Hz}$. The values of the storage modulus $\left(E^{\prime}\right)$, loss modulus $\left(E^{\prime \prime}\right)$ and loss factor $(\tan \delta)$ were obtained as a function of temperature. Relaxation temperatures were evaluated from the drop in $E^{\prime}$ curves and from the peaks in $E^{\prime \prime}$ and $\tan \delta$. The tensile tests were performed using a Zwick Z010 tensile test machine with a crosshead speed of $500 \mathrm{~mm} / \mathrm{min}$. Tensile test specimens with a gauge width of $3 \mathrm{~mm}$ and a gauge length of $30 \mathrm{~mm}$ were prepared by die cutting. The strain was determined by a Zwick video extensometer 066975 . The curves were averaged from 3 to 5 independent experiments. The tensile modulus was determined from the slope of the stress-strain curves using a linear fit to the data points within $10 \%$ strain. To evaluate the mechanical fatigue resistance, cyclic tests were performed on a TIRA test 2161 apparatus, Maschinenbau GmbH Ravenstein, Germany. Five cycles were run with a stationary time at maximum strength of $5 \mathrm{~s}$, stationary time at minimum strength of $5 \mathrm{~s}$, maximum strain of $100 \%$, and minimum strain of $8 \%$.

The complex dielectric permittivity at room temperature was recorded by sweeping frequency from 1 to $10^{6} \mathrm{~Hz}$, using a Novocontrol Dielectric Spectrometer equipped with frequency response analyzer. The samples were prepared by sputtering gold electrodes with a diameter of $20 \mathrm{~mm}$. The breakdown experiments were conducted with a setup similar to that described by Kollosche et al. [37]. About 10 measurements were done for each sample and the data were averaged. The electromechanical tests were performed using circular membrane actuators, for which the films were prestrained to about $12 \%$ and fixed between two circular frames. Circular electrodes (8 $\mathrm{mm}$ diameter) of carbon black powder were applied to each side of the film. A FUG HCL-35-12500 high voltage source served as power supply for actuator tests. The voltage was increased by $100 \mathrm{~V}$ every $2 \mathrm{~s}$ up to breakdown. The actuation strain was measured optically as the extension of the diameter of the electrode area via a digital camera, using an edge detection tool of a LabView program to detect the boundary between the black electrode area and the transparent silicone film.

\section{Synthesis of $P_{x}$}

Trimethylsilyl end-blocked silicones $\mathbf{P}_{\mathbf{x}}$, where $\mathbf{x}$ represents the mol\% of cyanopropyl groups were prepared according to [35] by hydrosilylation of poly(methylhydro)siloxane with allyl cyanide and 1-hexene in different proportions. $\mathbf{P}_{\mathbf{x}}$ have various content of cyanopropyl and hexyl groups as well as some unreacted hydrosilyl groups. The composition of $\mathbf{P}_{\mathbf{x}}$ is given in Table 1.

\begin{tabular}{|c|c|c|c|c|}
\hline $\begin{array}{l}\text { Polar } \\
\text { silicone }\end{array}$ & $\begin{array}{c}{ }^{\mathrm{a}} \text { Cyanopropylmethyl } \\
\text { siloxane unit } \\
{[\% \mathrm{~mol}]}\end{array}$ & $\begin{array}{c}{ }^{\mathrm{a}} \text { Hexylmethyl } \\
\text { siloxane unit } \\
{[\% \mathrm{~mol}]}\end{array}$ & $\begin{array}{l}{ }^{\mathrm{a}} \mathrm{H}-\mathrm{Si} \text { unit } \\
{[\% \mathrm{~mol}]}\end{array}$ & $\begin{array}{r}\varepsilon^{\prime} \text { at } \\
10 \mathrm{kHz}\end{array}$ \\
\hline$P_{89}$ & 89 & - & 11 & 15.9 \\
\hline$P_{62}$ & 62 & 26.9 & 11.1 & 11.3 \\
\hline$P_{10}$ & 10 & 78.7 & 11.3 & 3.7 \\
\hline$P_{0}$ & - & 98.5 & 1.5 & 2.7 \\
\hline
\end{tabular}

${ }^{\mathrm{a}}$ As determined by ${ }^{1} \mathrm{H}$ NMR spectroscopy. 


\section{Synthesis of materials $\mathbf{P}_{\mathbf{x}}(\mathbf{y} / \mathbf{z})$}

Interconnected networks between a polar $\mathbf{P}_{\mathbf{x}}$ component and a nonpolar PDMS matrix were prepared and named as $\mathbf{P}_{\mathbf{x}}(\mathbf{y} / \mathbf{z})$, where $\mathbf{P}_{\mathbf{x}}$ is the polar component used and $\mathrm{y} / \mathrm{z}$ indicate the mass ratio of $\mathbf{P}_{\mathbf{x}}$ to PDMS. The amount of reagents used for the synthesis of materials $\mathbf{P}_{\mathbf{x}}(\mathbf{y} / \mathbf{z})$ is given in Table 2 .

Below, the synthesis of $\mathbf{P}_{\mathbf{1 0}}(\mathbf{1 / 2})$ and its thin film formation is described:

(a) To a solution of copolymer $\mathbf{P}_{\mathbf{1 0}}(0.5 \mathrm{~g})$ in chloroform/methanol $1 / 1(2 \mathrm{~mL})$, DBTDL $(0.1 \mathrm{~mL})$ was added. The solution was stirred in an open vessel at room temperature. Chloroform was added when the solution became turbid, which indicates an excess of methanol (bad solvent). This procedure was repeated until no turbidity was observed, which took about $1 \mathrm{~h}$.

(b) To the resulting solution, TEOS $(0.8 \mathrm{~mL})$ was added and the mixture was stirred at $40^{\circ} \mathrm{C}$ for $15 \mathrm{~min}$.

(c) Finally, a solution of PDMS ( $1 \mathrm{~g})$ in chloroform was added and the mixture was stirred for about 5 min to obtain a homogenous solution. To remove the air bubbles this solution was sonicated for $1 \mathrm{~min}$. Films were made on a Teflon substrate using doctor blade technique.

Table 2: The amount of reagents used for the synthesis of materials $\mathbf{P}_{\mathbf{x}}(\mathbf{y} / \mathbf{z})$.

\begin{tabular}{ccccccc}
\hline Sample & $\begin{array}{c}\text { PDMS } \\
{[\mathrm{g}]}\end{array}$ & $\begin{array}{c}\mathbf{P}_{\mathbf{x}} \\
{[\mathrm{g}]}\end{array}$ & $\begin{array}{c}\text { TEOS } \\
{[\mathrm{mL}]}\end{array}$ & $\begin{array}{c}\text { Sn-cat. } \\
{[\mathrm{g}]}\end{array}$ & $\begin{array}{c}\mathbf{P}_{\mathbf{x}} \\
{[\mathrm{wt} . \%]}\end{array}$ & $\begin{array}{c}\text { CN group } \\
{[\mathrm{wt} \%]}\end{array}$ \\
\hline $\mathbf{P}_{\mathbf{8 9}}(\mathbf{1} / \mathbf{2})$ & 1 & 0.5 & 0.8 & 0.025 & 28.6 & 5.33 \\
$\mathbf{P}_{\mathbf{6 2}}(\mathbf{1} / \mathbf{2})$ & 1 & 0.50 & 0.80 & 0.1 & 27.0 & 3.33 \\
$\mathbf{P}_{\mathbf{1 0}}(\mathbf{1} / \mathbf{2})$ & 1 & 0.50 & 0.80 & 0.1 & 27.0 & 0.47 \\
$\mathbf{P}_{\mathbf{0}}(\mathbf{1} / \mathbf{2})$ & 1 & 0.50 & 0.80 & 0.1 & 27.0 & 0.00 \\
$\mathbf{P}_{\mathbf{8 9}}(\mathbf{1} / \mathbf{3})$ & 1.5 & 0.5 & 1.10 & 0.025 & 21.5 & 4.00 \\
$\mathbf{P}_{\mathbf{6 2}}(\mathbf{1} / \mathbf{3})$ & 1.5 & 0.50 & 0.80 & 0.1 & 21.5 & 2.73 \\
$\mathbf{P}_{\mathbf{1 0}}(\mathbf{1} / \mathbf{3})$ & 1.5 & 0.50 & 0.80 & 0.1 & 21.5 & 0.37 \\
$\mathbf{P}_{\mathbf{0}}(\mathbf{1} / \mathbf{3})$ & 1.5 & 0.50 & 0.80 & 0.1 & 21.5 & 0.00 \\
$\mathbf{P}_{\mathbf{6 2}}{ }^{\text {part }} \mathbf{( 1 / 2 )}$ & 0.3 & 0.15 & 0.12 & 0.025 & 28.4 & 3.50 \\
\hline
\end{tabular}

Synthesis of $P_{62}{ }^{\text {part }}(1 / 2)$

A mixture of copolymer $\mathrm{P}_{62}(0.15 \mathrm{~g})$ and one drop of DBTDL in a solution of methanol and THF $(1 / 1 \mathrm{v} / \mathrm{v})(1 \mathrm{~mL})$ was stirred in an open vessel for $30 \mathrm{~min}$. Then TEOS $(0.04 \mathrm{~mL})$ was added and the reaction mixture was stirred at $40{ }^{\circ} \mathrm{C}$ for another $10 \mathrm{~min}$. This solution was injected into a $1 \%$ solution of surfactant in toluene $(2 \mathrm{~mL})$. The methanol, THF and about half of toluene were removed under vacuum and the remaining solution was added to a solution of PDMS $(0.3 \mathrm{~g})$ in toluene $(1 \mathrm{~mL})$. Then TEOS $(0.08$ $\mathrm{mL}$ ) and one drop DBTDL were added followed by vigorous stirring for a few minutes. Films were prepared by casting on a Teflon substrate.

\section{Results and discussion}

1. Synthesis of polar-nonpolar interconnected elastic networks We have recently described the synthesis of a series of cyanopropyl/hexyl modified polysiloxanes $\mathbf{P}_{\mathbf{x}}$ with varying $\mathrm{mol} \%$ of polar cyano groups [35] by (co-)hydrosilylation reactions of a trimethylsilyl end-blocked poly(methylhydro)siloxane $\left(M_{w}\right.$ ca. 2000 $\mathrm{g} / \mathrm{mol}$ ) with a mixture of allyl cyanide and 1-hexene. An increase in the permittivity with increasing the amount of polar cyano groups from $\varepsilon^{\prime}=2.7$ for $\mathbf{P}_{0}$ to $\varepsilon^{\prime}=15.9$ for $\mathbf{P}_{89}$ (Table 1) was found. Because the molecular weight of $\mathbf{P}_{\mathbf{x}}$ was rather low, cross-linking of $\mathbf{P}_{\mathbf{x}}$ does not allow formation of materials with good elastic properties. However, the appropriate cross-linking of $\mathbf{P}_{\mathbf{x}}$ with a high $M_{w}$ PDMS might allow formation of materials with increased $\varepsilon^{\prime}$ and improved mechanical properties. Thus, it was the aim of this work to develop a synthetic strategy to interconnected $\mathbf{P}_{\mathbf{x}} /$ PDMS elastomers and to investigate how the amount of polar groups in $\mathbf{P}_{\mathbf{x}}$ influences the dielectric, mechanical, and electromechanical properties of the formed materials.

Although copolymers containing different mol\% of cyanopropyl groups can also be prepared starting from poly(dimethylmethylhydro)siloxane, several disadvantages can be noted when such copolymers are used. First, the poly(dimethylmethylhydro)siloxanes with varying content of methylhydrosiloxy groups have to be prepared starting from a mixture of $D_{4}$ and $D_{4} H$. This is time consuming, since the reaction conditions for each individual copolymer has to be first found. Furthermore, a systematic investigation of the influence of different cyanopropyl modified polymers on the properties of the resulting materials is not possible since they have different molecular weights and distributions. In addition, for trimethylsilyl-end blocked copolymers, the presence of a similar number of unreacted $\mathrm{Si}-\mathrm{H}$ groups should be ensured within a series for a systematic investigation. By using poly(methylhydro)siloxane and a mixture of allyl cyanide and 1hexene, polymers that have the same chain length and dispersity and tunable dielectric properties are formed. In this approach, 1hexene was a convenient liquid reagent used to ensure the same conversion within a series of copolymers with polar group content varying within a very wide range. Due to the low reactivity of allyl cyanide in the hydrosilylation, the conversion of the $\mathrm{Si}-\mathrm{H}$ was not quantitative (about $10 \mathrm{~mol} \%$ of $\mathrm{Si}-\mathrm{H}$ were still found in the final polymers). When only 1 -hexene was used for the hydrosilylation of poly(methylhydro)siloxane, the conversion of the $\mathrm{Si}-\mathrm{H}$ groups was almost quantitative $\left(\mathbf{P}_{0}\right)$. By using a three-step process, it was possible to incorporate a large amount of $\mathbf{P}_{\mathbf{x}}$ into PDMS, whereby the macroscopic phase separation was avoided. Materials $\mathbf{P}_{\mathbf{x}}(\mathbf{y} / \mathbf{z})$, where $y / z$ represents the weight ratio of $\mathbf{P}_{\mathbf{x}} /$ PDMS were prepared (Table 2). $\mathbf{P}_{\mathbf{0}}$ has no cyano groups and served as reference, while $\mathbf{P}_{\mathbf{8 9}}, \mathbf{P}_{\mathbf{6 2}}$ and $\mathbf{P}_{\mathbf{1 0}}$ were used as high $\varepsilon^{\prime}$ components. In the following, it will be shown how the unreacted $\mathrm{Si}-\mathrm{H}$ groups of $\mathbf{P}_{\mathbf{x}}$ were used to anchor $\mathbf{P}_{\mathbf{x}}$ to the PDMS matrix.

In our previous work, we observed that when a hydroxyl endfunctionalized poly(dimethyl-methylhydro)siloxane was hydrosilylated with allyl cyanide, a spontaneous cross-linking of copolymers occurred [34]. It was presumed that a condensation between the hydroxyl end-groups and the unreacted residual $\mathrm{Si}-\mathrm{H}$ groups catalyzed by Pt occurred. Our attempts to avoid this side reaction by replacing the hydroxyl end-groups by trimethylsilyl were not successful [35]. When $\mathbf{P}_{\mathbf{x}}$ or their solutions in toluene were kept in normal atmosphere, an insoluble cross-linked material formed. The ${ }^{29} \mathrm{Si}$ NMR spectrum of this cross-linked material swollen in $\mathrm{CDCl}_{3}$ shows the presence of the " $\mathrm{T}$ "-type $\mathrm{Si}$ formed from two $\mathrm{Si}-\mathrm{H}$ groups 
via a hydrolysis followed by a condensation step (Fig. 1S). Although at first hand this was disappointing, we decided to use this sidereaction in our advantage. The FT-IR spectra of these cross-linked materials show that the vibration band at $2154 \mathrm{~cm}^{-1}$ typical for $\mathrm{Si}-\mathrm{H}$ groups disappeared after few months. It is known that the $\mathrm{Si}-\mathrm{H}$ bond hydrolyses slowly in presence of moisture and fast in presence of bases [26]. The cross-linking of $\mathbf{P}_{\mathbf{x}}$ is most likely due to the hydrolysis of the $\mathrm{Si}-\mathrm{H}$ groups to $\mathrm{Si}-\mathrm{OH}$ followed by a spontaneous dehydration between two $\mathrm{Si}-\mathrm{OH}$ groups and/or a dehydrocoupling between the formed $\mathrm{Si}-\mathrm{OH}$ and the remaining $\mathrm{Si}-\mathrm{H}$ groups. The latter reaction is favored by the presence of traces of Pt or Sn catalyst. Based on these observations, a three-step process for the synthesis of polar-nonpolar interconnected elastic networks was developed (Scheme 1):

(a) The hydrolysis of $\mathrm{Si}-\mathrm{H}$ groups of $\mathbf{P}_{\mathbf{x}}$ in a solution of chloroformmethanol in the presence of DBTDL and atmospheric moisture.

(b) The hydrolysis of TEOS and condensation of the formed $\mathrm{Si}-\mathrm{OH}$ groups to generate silica in-situ and to anchor $\mathbf{P}_{\mathbf{x}}$.

(c) The condensation of the hydroxyl end-groups of PDMS with the hydroxyl groups of the polar component obtained in step b).

As these condensation reactions are rather slow, maturation in time is necessary as a final step.
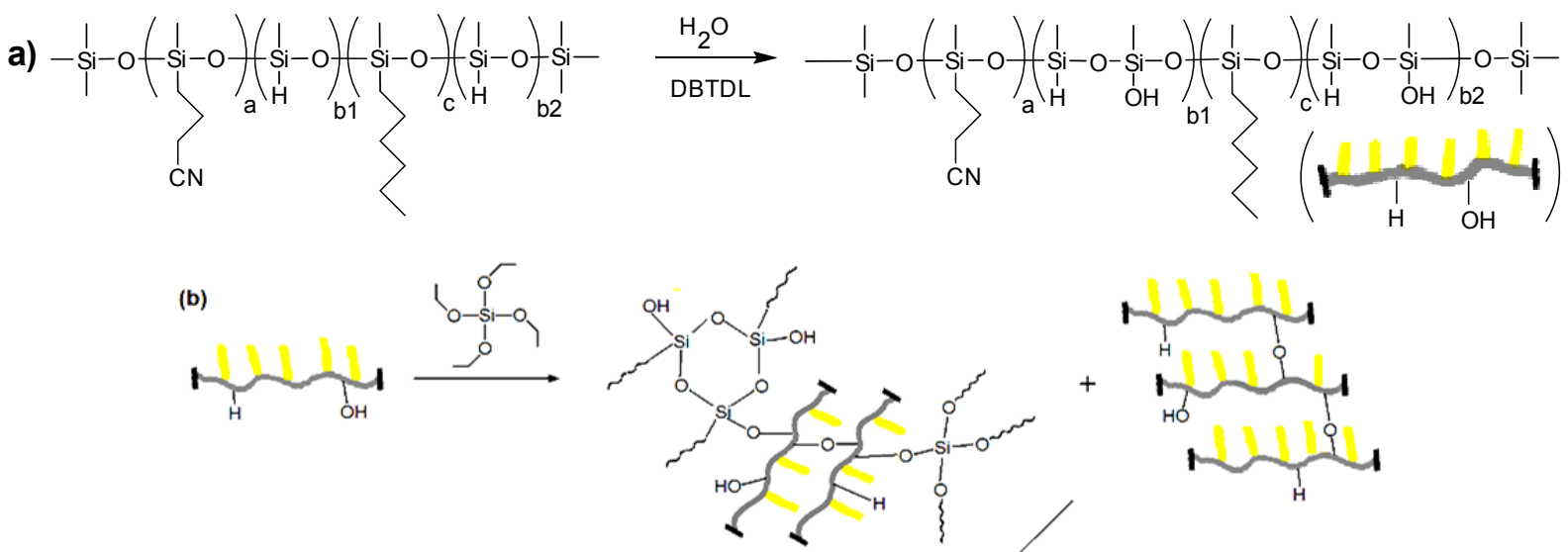

(c)

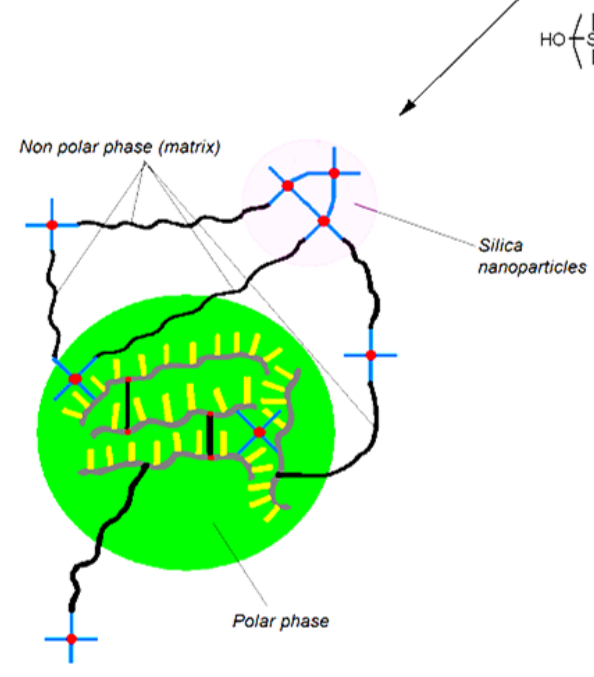

Scheme 1: Schematic illustration of the three-step process used for the formation of polar-nonpolar interconnected silicone elastomers.

To monitor the evolution of the reaction stepwise, a kinetic survey at various moments of the process was done by FT-IR. The changes of a solution of $\mathbf{P}_{62}$ in a mixture of chloroform-methanol in the presence of DBTDL were observed. Aliquots were taken from time to time and the changes in the $\mathrm{Si}-\mathrm{H}$ stretching band at $2154 \mathrm{~cm}^{-1}$ were followed while the $\mathrm{Si}-\mathrm{CH}_{3}$ symmetric band at $1260 \mathrm{~cm}^{-1}$ was taken as reference (Fig. 2S). After about $1 \mathrm{~h}$, the $\mathrm{Si}-\mathrm{H}$ band decreased to half. The residual $\mathrm{Si}-\mathrm{H}$ groups are sterically hindered and therefore less reactive. Additionally, the vicinity of hydrophobic methylsiloxane groups makes the hydrolysis more difficult. It is therefore not surprising that this hydrolysis step is rather slow. As mentioned before, $\mathbf{P}_{89}, \mathbf{P}_{\mathbf{6 2}}$ and $\mathbf{P}_{10}$ contain around $10 \%$ un-modified $\mathrm{Si}-\mathrm{H}$ groups which represent 3.3 groups in average per polymer chain. After $1 \mathrm{~h}$, about $50 \%$ of these groups were hydrolyzed. In principle, even if only a single $\mathrm{Si}-\mathrm{H}$ group per copolymer chain would react, this would be sufficient to anchor $\mathbf{P}_{\mathbf{x}}$ to the PDMS but does not ensure its use as sole cross-linker for PDMS in a 3D network. If the reaction time is increased, the condensation process occurs simultaneously, leading to self-cross-linking of $\mathbf{P}_{\mathbf{x}}$.

Therefore, the total cross-linking capacity of the polar phase was increased by adding TEOS in the second step, which easily hydrolyzes in atmosphere. The FT-IR spectra (350-1300 $\mathrm{cm}^{-1}$ region) of the hydrolysis and condensation processes in steps (a) and (b) 
are compared with that of the polar copolymer $\mathbf{P}_{62}$ in Fig. 1. The starting $\mathbf{P}_{62}$ shows the well-known $\mathrm{Si}-\mathrm{O}-\mathrm{Si}$ absorption pattern consisting of two broad and strong symmetric bands at 1021 and $1096 \mathrm{~cm}^{-1}$, respectively. After the first step, this region of the spectrum changed. Three bands were present at 1015, 1085 and $1110 \mathrm{~cm}^{-1}$ and an asymmetric pattern was observed. This asymmetry is specific to so-called "hydrolyzed" trifunctional siloxanes [38]. Apart from the modifications of the siloxane region, the spectrum of the hydrolyzed $\mathbf{P}_{62}$ shows an absorption band at $966 \mathrm{~cm}^{-1}$ characteristic for Si-OH groups [38] which is not present in the starting copolymer. After TEOS addition, the condensation reaction started. The spectrum shows smoothening of the asymmetric pattern and a broadening of the entire siloxane region $\left(1000-1130 \mathrm{~cm}^{-1}\right)$. These important changes in the spectrum indicate the formation of a 3D silica-like network. Additionally, the stretching band at $966 \mathrm{~cm}^{-1}$ characteristic to the $\mathrm{Si}-\mathrm{OH}$ bond remains as a shoulder.

In the third step the condensation of the hydroxyl end-groups of PDMS with the hydroxyl groups of the polar component occurred. Thin films were then formed by doctor blade technique on a Teflon substrate. After evaporation of the solvent and finalization of the condensation process, the films were peeled off the substrate. The un-hydrolyzed $\mathrm{Si}-\mathrm{H}$ groups that were still observed in the crosslinked film slowly hydrolyzed over time under atmospheric humidity; however this process is slow and can take weeks or even months. An example is shown in Fig.3S, where the gradual decrease of the $\mathrm{Si}-\mathrm{H}$ absorbance within three weeks was observed. Therefore, the film formation process requires maturation. Although a simple procedure is used, the cross-linking in the film is a rather complex process, which involves hydrolysis and condensation reactions which finally lead to interconnected networks. The condensation reactions can occur between the hydroxyl groups formed by hydrolysis of TEOS, those present in $\mathbf{P}_{\mathbf{x}}$ after step (a) and the hydroxyl end-groups from PDMS matrix (Scheme 1).

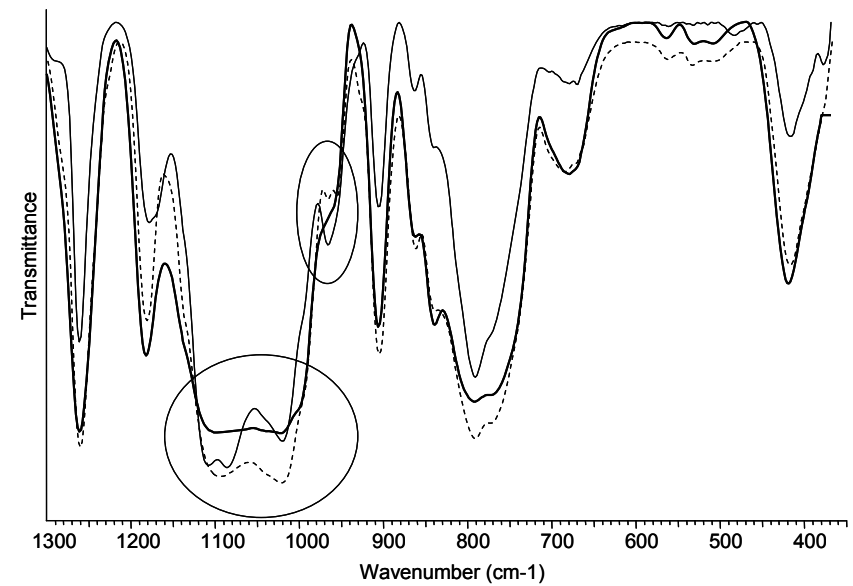

Fig. 1. Comparison of FT-IR spectra (350-1300 $\mathrm{cm}^{-1}$ region) of the polar copolymer component at different stages: $\mathbf{P}_{\mathbf{6 2}}$ just after the synthesis (dotted line), after the hydrolysis-step (a) (thin line), and after the condensation-step (b) (bold line).
Due to the remaining Si-H groups, one may think that the polar $\mathbf{P}_{\mathbf{x}}$ could be used alone to cross-link the PDMS. However, according to literature [31] and our own experiments, the hydrosilylation reaction in this particular case was not successful, probably due to steric hindrance. Moreover, if $\mathbf{P}_{\mathbf{x}}$ is used as cross-linker, stoichiometric amounts would be used, which would allow incorporation of only about 3 wt $\%$ of polar component $\mathbf{P}_{\mathbf{x}}$ into the materials. This amount of polar $\mathbf{P}_{\mathbf{x}}$ component would be too low to induce a significant increase in the permittivity of the PDMS matrix. Furthermore, if excess of polar $\mathbf{P}_{\mathbf{x}}$ is used without ensuring conditions for its cross-linking during film formation, the polar phase is not chemically bonded to the PDMS matrix and therefore macrophase separated materials form (an example is shown in Fig. 4S). By using TEOS which functions as an interconnecting agent between the polar and nonpolar components, the phase separation can be controlled. Additionally, the excess of TEOS allows in-situ formation of silica, which reinforces the materials.

The amount of extractable species in THF was about $8 \%$ for the materials $\mathbf{P}_{\mathbf{6 2}} \mathbf{( 1 / 2 )}$. ${ }^{1} \mathrm{H}$ NMR spectrum (Fig. $5 \mathrm{~S}$ ) shows that a mixture of compounds: DBTDL catalyst, some oligodimethylsiloxane and $\mathbf{P}_{\mathbf{6 2}}$ was extracted. According to the ${ }^{1} \mathrm{H}$ NMR spectrum, the amount of $\mathbf{P}_{62}$ was ca. $7 \%$ in the extract, which represents about $0.5 \% \mathbf{P}_{62}$ that is not interconnected into the network. Since the characteristic signal for $\mathrm{Si}-\mathrm{H}$ protons is not present in the ${ }^{1} \mathrm{H}$ NMR spectrum of the extract, it might be that a small fraction of $\mathbf{P}_{62}$ had a complete conversion of the $\mathrm{Si}-\mathrm{H}$ groups in the hydrosilylation.

\section{Characterization of the films}

SEM investigations conducted on cryo-fractured films, clearly showed phase separation with globular domains. Fig. 2 shows four representative SEM images of materials $\mathbf{P}_{\mathbf{8 9}}(\mathbf{1 / 2}), \mathbf{P}_{6 \mathbf{2}}(\mathbf{1} / \mathbf{2}), \mathbf{P}_{\mathbf{1 0}}(\mathbf{1} / \mathbf{2})$, and $P_{\mathbf{0}}(\mathbf{1 / 2})$. Phase separation is a common phenomenon in poly(dimethylsiloxane) copolymers or blends [39, 40], since PDMS is incompatible with practically any organic polymer. As a consequence of this incompatibility, phase separation was observed not only for the highly polar copolymers $\mathbf{P}_{\mathbf{8 9}}, \mathbf{P}_{\mathbf{6 2}}$ and $\mathbf{P}_{\mathbf{1 0}}$ but also for $\mathbf{P}_{\mathbf{0}}$ which contains long aliphatic branches. However, an interesting aspect is that the morphology is uniform over the entire thickness of the film and no layering was observed. When the polar copolymer was blended in PDMS without being cross-linked, the resulting material had a completely different aspect, with macroscopic phase separation (Fig. 4S), due to the different polarity and density of the components. Therefore, the morphology observed for $\mathbf{P}_{\mathbf{x}}(\mathbf{y} / \mathbf{z})$ films supports the idea of interconnected networks. 

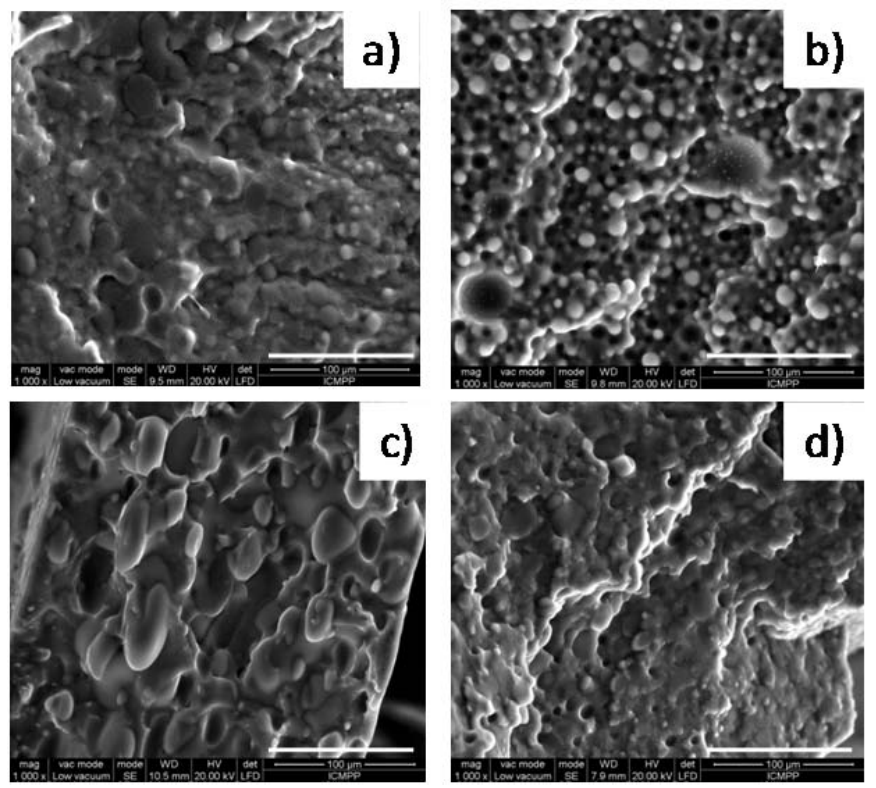

Fig. 2 SEM images of the cryo-fractured films: $\left.\mathbf{P}_{89}(\mathbf{1 / 2})-a\right), \mathbf{P}_{\mathbf{6 2}}(\mathbf{1 / 2})$ b), $\left.\mathbf{P}_{\mathbf{1 0}}(\mathbf{1 / 2})-c\right)$, and $\left.\mathbf{P}_{\mathbf{0}}(\mathbf{1 / 2})-d\right)$. Size bar represents $100 \mu \mathrm{m}$.

To further reduce the polar domains in our materials, particles of cross-linked $\mathbf{P}_{62}$ were prepared by precipitation technique as described in the experimental part. These particles were incorporated in a solution of PDMS and cross-linking reagents in toluene under stirring. A film of the above mixture was casted on a Teflon substrate (Sample $\mathbf{P}_{\mathbf{6 2}}{ }^{\text {part }} \mathbf{( 1 / 2 )}$ ). The SEM image of this material (Fig. 6S) showed that submicron particles are dispersed within the matrix, which represents a reduction of the polar domains roughly by a factor of 10 . However, $\mathbf{P}_{\mathbf{6 2}}{ }^{\text {part }}(\mathbf{1} / \mathbf{2})$ is a blend, since the polar and nonpolar phases are not chemically bonded.

The DSC analysis of the interconnected networks revealed two glass transition temperatures $\left(T_{g}\right)$ irrespective of the composition of the films (Table 3 ). The presence of two $T_{g}$ s indicates that the materials are biphasic. The lower value $T_{g} 1$ corresponds to the nonpolar silicone phase and is generally higher than in neat PDMS ($124.5^{\circ} \mathrm{C}$ ), while the other value, $T_{g} 2$, is assigned to the respective polar component.

The absolute heat capacity characterizes energetically the movement of chain segments. Cross-linking leads to reduction in free volume between chain segments. In a cross-linked material, the movement of the chain segments is sterically hindered, which lowers the absolute heat capacity values [41, 42]. In our case, $\Delta \mathrm{Cp}=$ $0.306 \mathrm{~J} / \mathrm{gK}$ for the pristine, un-cross-linked PDMS. The content of PDMS in our materials, calculated by taking into account all the components (PDMS, polar silicone, catalyst and silica resulted from full hydrolysis and condensation of the excess TEOS) is ca. $55 \%$ for $\mathbf{P}_{\mathbf{x}}(\mathbf{1} / \mathbf{2})$ films and ca. $65 \%$ for $\mathbf{P}_{\mathbf{x}}(\mathbf{1} / \mathbf{3})$ films. The theoretical values for $\Delta \mathrm{Cp}(1)$ in the films were: $0.165 \mathrm{~J} / \mathrm{gK}$ for $\mathbf{P}_{\mathrm{x}}(\mathbf{1} / \mathbf{2})$ and $0.2 \mathrm{~J} / \mathrm{gK}$ for $P_{x}(\mathbf{1} / 3)$. The theoretical $\Delta C p$ values for $T_{g} 2$ were also calculated (column 5 in Table 3 ), from $\Delta \mathrm{Cp}$ of the corresponding un-crosslinked copolymer (second column) and the composition of the films. As can be observed in Table 3, the experimental $\Delta C p$ values of the new materials for both $T_{g}$ s are lower as compared to the calculated values. This indicates that the macromolecular chains have diminished mobility, which supports the cross-linked state of PDMS and of $\mathbf{P}_{\mathbf{x}}$ in the films. As an example, Fig.7S compares the DSC curve of $P_{10}(\mathbf{1} / \mathbf{2})$ with that of $P_{10}$ and PDMS.

Table 3: Thermal behavior of $\mathbf{P}_{\mathbf{x}}(\mathbf{y} / \mathbf{z})$ as determined by DSC.

\begin{tabular}{|c|c|c|c|c|c|}
\hline \multirow[t]{2}{*}{ Film } & \multirow{2}{*}{$\begin{array}{l}T_{g} \text { of } \mathbf{P}_{\mathbf{x}},{ }^{\circ} \mathrm{C} \\
(\Delta \mathrm{Cp}, \mathrm{J} / \mathrm{gK})\end{array}$} & \multicolumn{3}{|c|}{$T_{g} \mathrm{~s}$ in film $\left({ }^{\circ} \mathrm{C}\right)$} & \multirow[t]{2}{*}{$T_{m}\left({ }^{\circ} \mathrm{C}\right)$} \\
\hline & & $\begin{array}{c}T_{g} 1 \\
(\Delta \mathrm{Cp}, \mathrm{J} / \mathrm{gK})\end{array}$ & $\begin{array}{c}T_{g}^{2} \\
(\Delta \mathrm{Cp}, \mathrm{J} / \mathrm{gK})\end{array}$ & $\begin{array}{l}\text { Calcd. } \Delta \mathrm{Cp}(2)^{*} \\
\text { J/gK }\end{array}$ & \\
\hline$P_{89}(1 / 2)$ & $-62.1(0.338)$ & $-123.6(0.027)$ & $-63.2(0.051)$ & 0.097 & -42 \\
\hline$P_{89}(1 / 3)$ & & $-121.8(0.029)$ & $-60.6(0.039)$ & 0.073 & -40 \\
\hline$P_{62}(1 / 2)$ & $-75.6(0.344)$ & $-118(0.045)$ & $-88(0.08)$ & 0.092 & -45 \\
\hline$P_{62}((1 / 3)$ & & $-117(0.034)$ & $-88.4(0.06)$ & 0.075 & -44 \\
\hline$P_{10}(1 / 2)$ & $-103(0.43)$ & $-117(0.005)$ & $-95(0.054)$ & 0.116 & -44 \\
\hline$P_{10}(1 / 3)$ & & $-122(0.032)$ & $-108(0.08)$ & 0.092 & -44 \\
\hline$P_{0}(1 / 2)$ & $-111.3(0.405)$ & $-121(0.081)$ & $-111(0.063)$ & 0.11 & $-43.5 ;-9$ \\
\hline$P_{0}(1 / 3)$ & & $-119(0.038)$ & $-111(0.072)$ & 0.087 & $-42.3 ;-9$ \\
\hline
\end{tabular}

${ }^{*}$ Calculated according to the composition, with the formula: $\% \mathbf{P}_{\mathbf{x}} \cdot \Delta \mathrm{Cp}\left(\mathbf{P}_{\mathbf{x}}\right)$

During the heating scan, a melting endotherm was observed between -42 and $-45{ }^{\circ} \mathrm{C}$, but no cold crystallization (as observed for PDMS - Fig. 7S). The formation of crystalline domains was registered during the cooling scan between -75 and $-73^{\circ} \mathrm{C}$ (not shown). These transitions were assigned to the nonpolar component since their values are close to the values of neat PDMS, i.e. $-44{ }^{\circ} \mathrm{C}$ melting (heating scan) and $-82{ }^{\circ} \mathrm{C}$ crystallization (cooling) and neither melting nor crystallization phenomena were detected for $\mathbf{P}_{\mathbf{x}}$. For materials $\mathbf{P}_{\mathbf{0}}(\mathbf{y} / \mathbf{z})$ additional transitions were observed: melting at around $-9{ }^{\circ} \mathrm{C}$ (heating scan) and crystallization at -37 to $40{ }^{\circ} \mathrm{C}$ (cooling scan). Since $\mathbf{P}_{\mathbf{0}}$ didn't exhibit such transitions, they might be due to differently organized domains in PDMS, as suggested by Sundarajan [43]. On the other hand, we cannot exclude that crystallization of the poly(methylhexyl)siloxane might be favored in the cross-linked materials. As mentioned before, polymer $\mathbf{P}_{\mathbf{0}}$ contains only few unreacted $\mathrm{Si}-\mathrm{H}$ groups. Following the rational of the cross-linking process, described above, it is unlikely for materials $\mathbf{P}_{0}(\mathbf{y} / \mathbf{z})$ to cross-linking via $\mathbf{P}_{\mathbf{0}}$. Despite of this, no macroscopic phase separation or exudation was observed. In addition, even though the $T_{g} 2$ value is close to that of the neat polymer, the corresponding $\Delta C p$ values are lower than the calculated ones. This is an indication that the chains participate in 
the cross-linking reaction during film formation which limits chain motion.

Material $\mathbf{P}_{\mathbf{6 2}} \mathbf{( 1 / 2 )}$ was analyzed by DMA. Its viscoelastic behavior is characterized by a glassy region $\left(T>-140{ }^{\circ} \mathrm{C}\right)$ with a storage modulus in the order of $10^{9} \mathrm{~Pa}$, followed by a two steps decrease of the storage modulus up to $-50{ }^{\circ} \mathrm{C}$ (Fig. 8S). The decrease of the storage modulus is less than one order of magnitude in the $T_{g}$ region and the low value of $\tan \delta$ peak height indicate the presence of constraints that hinder the large movement of chain segments. These constrains are on one hand the cross-linking points, and on the other hand, the crystalline domains confirmed by the DSC measurements. At $-38{ }^{\circ} \mathrm{C}$ an abrupt descent in $E^{\prime}$, more than three orders of magnitude over a narrow temperature range was observed, until a plateau marks the melting of the crystalline phase. The storage modulus reaches a plateau that has the magnitude of $10^{4} \mathrm{~Pa}$, which is comparable with the values previously obtained for medium weight PDMS-silica cross-linked films [44]. The low value of the storage modulus indicates that soft materials are formed.

The mechanical properties of $\mathbf{P}_{\mathbf{x}}(\mathbf{y} / \mathbf{z})$ and a reference crosslinked PDMS film obtained with the same ratio of cross-linking reagents, were evaluated in tensile tests. The stress-strain curves

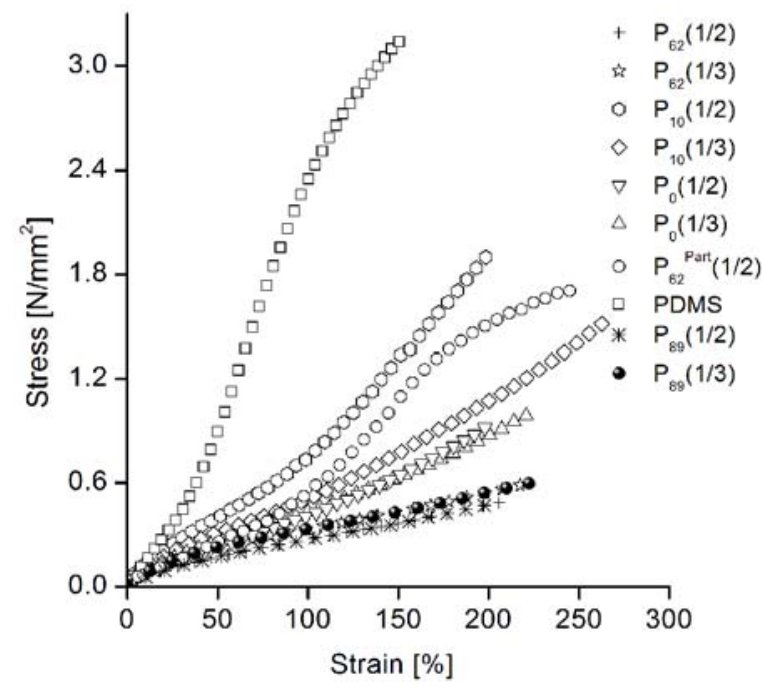

Fig. 3 Stress-strain curves of $\mathbf{P}_{\mathbf{x}}(\mathbf{y} / \mathbf{z})$ and PDMS film.

Cyclic tests were performed on samples $\mathbf{P}_{\mathbf{6 2}}(\mathbf{1 / 2})$ and $\mathbf{P}_{\mathbf{1 0}}(\mathbf{1 / 2})$ to evaluate the mechanical fatigue resistance. The tests prove the elastic behavior of the elastomer films, with a hysteresis loop only at the first strain-release cycle and a small difference between cycles (Fig. 9S).

The dielectric properties of the cross-linked films were investigated by dielectric relaxation spectroscopy at room temperature between 1 and $10^{6} \mathrm{~Hz}$. Fig. 4 shows the variation in $\varepsilon^{\prime}$, dielectric loss $\left(\varepsilon^{\prime \prime}\right)$, loss factor (tan $\delta$ ) and conductivity $(\sigma)$ for materials $P_{x}(y / z)$ and the PDMS reference film. Table 5 summarizes are represented in Fig. 3, while in Table 4 the Young's moduli, maximum stress and deformation are collected. A large excess of TEOS was used in all the films, including the PDMS reference, i.e. ca. $50 \mathrm{wt} \%$ reported to the polymers. It is therefore not surprising that our PDMS matrix (reference) is rather stiff with a maximum strain of $154 \%$ and a maximum stress of $3.2 \mathrm{~N} / \mathrm{m}^{2}$. Materials $\mathbf{P}_{\mathbf{x}}(\mathbf{y} / \mathbf{z})$ were softer than the PDMS reference. The lowest value for maximum strain was $170 \%$ for $\mathbf{P}_{\mathbf{6 2}}(\mathbf{1 / 2})$, while all the other samples gave values between 200 and $270 \%$. The obtained materials have Young's modulus at $10 \%$ strain that ranges between 0.4 and 1.5 $\mathrm{MPa}$, and maximum stress roughly between 0.5 and $2 \mathrm{MPa}$. Materials $\mathbf{P}_{\mathbf{x}}(\mathbf{1 / 2})$ have a lower strain at break as compared to materials $\mathbf{P}_{\mathbf{x}} \mathbf{( 1 / 3 )}$. The mechanical properties of material $\mathbf{P}_{\mathbf{6 2}}{ }^{\text {part }} \mathbf{( 1 / 2 )}$ were different from those of $\mathbf{P}_{\mathbf{6 2}}(\mathbf{1} / \mathbf{2})$ that has the same amount of polar phase incorporated, i.e. the tensile strength increased significantly, the strain at break also increased (to $250 \%$, from $170 \%$ ), and $Y$ decreased to $0.48 \mathrm{MPa}$ (from $0.8 \mathrm{MPa}$ ).

A plasticizing effect of $\mathbf{P}_{\mathbf{x}}$ on the PDMS matrix was observed for all the interconnected networks. Such effect was reported before for blends and was assigned to the additional volume of the dispersed phase $[31,45]$, when the phases were mixed in solution.

Table 4: Mechanical properties of $\mathbf{P}_{\mathbf{x}}(\mathbf{y} / \mathbf{z})$ and PDMS reference film

\begin{tabular}{|c|c|c|c|c|c|c|}
\hline \multirow{2}{*}{ Sample } & \multicolumn{4}{|c|}{ Young's modulus (MPa) } & \multirow{2}{*}{$\begin{array}{l}\text { Tensile } \\
\text { strength } \\
{[\mathrm{MPa}]}\end{array}$} & \multirow{2}{*}{$\begin{array}{l}\text { Elongation } \\
\text { at break } \\
\text { [\%] }\end{array}$} \\
\hline & $10 \%$ & $20 \%$ & $50 \%$ & $100 \%$ & & \\
\hline$P_{89}(1 / 2)$ & 0.43 & 0.57 & 0.46 & 0.55 & 0.49 & 206 \\
\hline$P_{89}(1 / 3)$ & 0.65 & 0.47 & 0.26 & 0.53 & 0.60 & 225 \\
\hline$P_{62}(1 / 2)$ & 0.80 & 0.69 & 0.52 & 0.67 & 1.07 & 170 \\
\hline$P_{62}(1 / 3)$ & 0.75 & 0.62 & 0.41 & 0.39 & 1.02 & 208 \\
\hline$P_{10}(1 / 2)$ & 0.89 & 0.75 & 0.57 & 0.87 & 1.88 & 200 \\
\hline$P_{10}(1 / 3)$ & 0.71 & 0.59 & 0.38 & 0.44 & 1.52 & 266 \\
\hline$P_{0}(1 / 2)$ & 0.47 & 0.42 & 0.34 & 0.39 & 0.88 & 199 \\
\hline$P_{0}(1 / 3)$ & 0.52 & 0.46 & 0.33 & 0.37 & 0.99 & 227 \\
\hline PDMS & 1.37 & 1.32 & 2.82 & 2.27 & 3.20 & 154 \\
\hline$P_{62}{ }^{\text {part }}(1 / 2)$ & 0.48 & 0.45 & 0.46 & 0.70 & 1.71 & 247 \\
\hline
\end{tabular}

the dielectric properties at $10 \mathrm{kHz}$. Compared to the reference PDMS film $\left(\varepsilon^{\prime}=2.6\right)$, all materials $P_{x}(y / z)$ show an increase in permittivity, up to $\varepsilon^{\prime}=4.9$ for $\mathrm{P}_{89}(1 / 2)$. The conductivity values at low frequencies are between $5 \times 10^{-12}$ and $5 \times 10^{-11} \mathrm{~S} \mathrm{~cm}^{-1}$, while the dielectric losses at high frequencies are rather low. Materials $\mathrm{P}_{89}(\mathrm{y} / \mathrm{z})$ and $\mathrm{P}_{62}(\mathrm{y} / \mathrm{z})$ show a relaxation peak between $50 \mathrm{~Hz}$ and 1 $\mathrm{kHz}$ which results in an increase in the $\varepsilon^{\prime}$. This relaxation peak might be due to interfacial polarization which occurs at low frequencies [46]. A $\varepsilon^{\prime}$ increase at low frequencies was observed for all samples which is most likely due to electrode polarization. 


\section{Journal Name}

\section{ARTICLE}

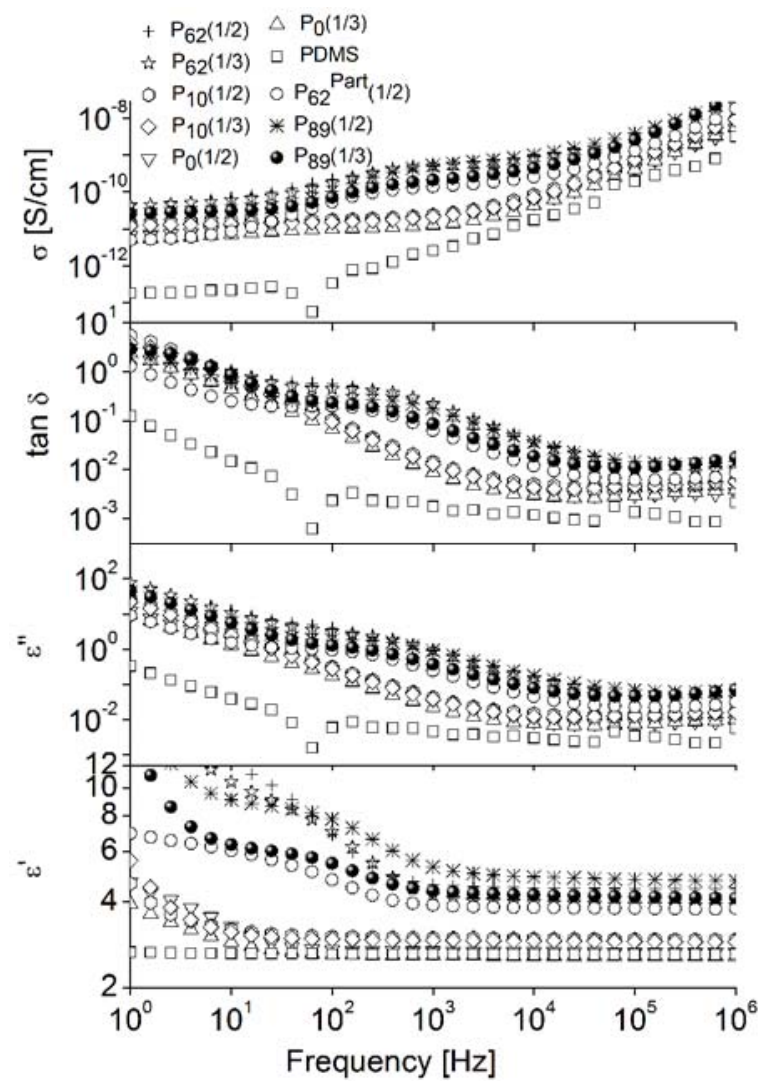

Fig. 4 Dielectric permittivity $\left(\varepsilon^{\prime}\right)$, dielectric loss $\left(\varepsilon^{\prime \prime}\right)$, loss factor (tan $\delta)$ and conductivity $(\sigma)$ of $\mathrm{P}_{\mathrm{x}}(\mathrm{y} / \mathrm{z})$ and of the PDMS matrix as function of the frequency.

Table 5: Dielectric properties, actuation strain $(s)$ at $30 \mathrm{~V} / \mu \mathrm{m}$, maximum actuation strain $\left(s_{\max }\right)$, the dielectric breakdown in actuator $\left(E_{b}\right)$, and the dielectric strength $\left(E_{\mathrm{EB}}\right)$ for $\mathbf{P}_{\mathbf{x}}(\mathbf{y} / \mathbf{z}), \mathbf{P}_{\mathbf{6 2}}{ }^{\text {part }}(\mathbf{1 / 2})$ and PDMS reference.

\begin{tabular}{|c|c|c|c|c|c|c|c|}
\hline Sample & $\begin{array}{c}\varepsilon^{\prime} \\
(10 \mathrm{kHz})\end{array}$ & $\begin{array}{c}\varepsilon^{\prime \prime} \text { at } \\
(10 \mathrm{kHz})\end{array}$ & $\begin{array}{c}\sigma(10 \mathrm{kHz}) \\
{\left[\mathrm{S} \mathrm{cm}^{-1}\right]}\end{array}$ & $\begin{array}{c}s[\%] \\
\text { at } 30 \mathrm{~V} / \mu \mathrm{m}\end{array}$ & $s_{\max }[\%]$ & $\begin{array}{c}E_{b}^{a} \\
{[\mathrm{~V} / \mu \mathrm{m}]}\end{array}$ & $\begin{array}{c}E_{\mathrm{EB}}^{\mathrm{b}} \\
{[\mathrm{V} / \mu \mathrm{m}]}\end{array}$ \\
\hline$P_{89}(1 / 2)$ & 4.9 & 0.177 & $9.9 \times 10^{-10}$ & - & 1.7 & 19 & 29 \\
\hline$P_{89}(1 / 3)$ & 4.2 & 0.079 & $4.4 \times 10^{-10}$ & - & 1.5 & 25 & 34 \\
\hline$P_{62}(1 / 2)$ & 4.5 & 0.095 & $5.3 \times 10^{-10}$ & 1.9 & 8 & 56 & 61 \\
\hline$P_{62}(1 / 3)$ & 4 & 0.145 & $8.1 \times 10^{-10}$ & 2.2 & 3.2 & 34 & 58 \\
\hline$P_{10}(1 / 2)$ & 3 & 0.015 & $8.3 \times 10^{-11}$ & 1.1 & 5.6 & 59 & 61 \\
\hline$P_{10}(1 / 3)$ & 2.9 & 0.012 & $6.9 \times 10^{-11}$ & 1.3 & 3.3 & 47 & 71 \\
\hline$P_{0}(1 / 2)$ & 2.7 & 0.009 & $5.2 \times 10^{-11}$ & 3.5 & 4.4 & 34 & 54 \\
\hline$P_{0}(1 / 3)$ & 2.5 & 0.008 & $4.3 \times 10^{-11}$ & 2.1 & 2 & 31 & 62 \\
\hline$P_{62}{ }^{\text {part }}(1 / 2)$ & 3.8 & 0.045 & $2.2 \times 10^{-10}$ & 3 & 3.6 & 31.5 & 65.4 \\
\hline PDMS & 2.6 & 0.003 & $1.7 \times 10^{-11}$ & 0.3 & 0.6 & 49 & 128 \\
\hline
\end{tabular}

${ }^{\mathrm{a}} E_{b}$ for actuator measurements using a carbon black electrode with a diameter of $8 \mathrm{~mm}$;

${ }^{\mathrm{b}} E_{E B}$ of the material using $1 \mathrm{~mm}^{2}$ electrodes [37]. 


\section{Electromechanical performances}

The new materials were also investigated in electromechanical tests using circular actuators. An increasing voltage step was applied to the samples until a breakdown through the material occurred. The measured actuation strains of the materials are shown in Fig. 5 and compared with the PDMS reference. Table 5 summarizes the maximum actuation strain, the breakdown field of the actuator, the dielectric breakdown, and the actuation strains at $30 \mathrm{~V} / \mu \mathrm{m}$ for all materials. The largest lateral actuation strain at $30 \mathrm{~V} / \mu \mathrm{m}$ was obtained for material $\mathbf{P}_{\mathbf{0}} \mathbf{( 1 / 2 )}(\mathbf{3} .5 \%)$ which was the softest of the materials that withstand this field, while the highest maximum lateral actuation strain of $8 \%$ was obtained for $\mathbf{P}_{62}(\mathbf{1 / 2})$ at $56 \mathrm{~V} / \mu \mathrm{m}$. The obtained strain values are roughly 12 or 13 -times higher as compared to the PDMS reference. The highest actuation strain at the lowest electric field was observed form material $\mathbf{P}_{\mathbf{8 9}} \mathbf{( 1 / 2 )}$ with a $s_{\max }=1.6 \%$ at $18.5 \mathrm{~V} / \mu \mathrm{m}$.

Irrespective of the type of polar polymer $\mathbf{P}_{\mathbf{x}}$ used, materials $P_{x}(\mathbf{1} / \mathbf{2})$ show an increase in the breakdown field and an increase in the maximum achievable lateral strain as compared to materials $P_{\mathbf{x}}(\mathbf{1 / 3})$. Material $\mathbf{P}_{\mathbf{8 9}}(\mathbf{1 / 2})$ has slightly lower breakdown field in actuator but a larger actuation strain at a certain voltage as compared to $\mathbf{P}_{\mathbf{8 9}}(\mathbf{1 / 3})$. Material $\mathbf{P}_{\mathbf{6 2}}(\mathbf{1 / 2})$ has a maximum actuation strain $\left(s_{\max }\right)$ of $8 \%$ at $E_{b}=56 \mathrm{~V} / \mu \mathrm{m}$, while material $\mathbf{P}_{62}(\mathbf{1} / 3)$ has a much lower $s_{\max }\left(s_{\max }=3.2 \%\right)$ at $E_{b}=34 \mathrm{~V} / \mu \mathrm{m}$. The same trend was observed for sample $\mathbf{P}_{\mathbf{1 0}}(\mathbf{1 / 2})$ with a $s_{\max }=5.6 \%$ at $E_{b}=59 \mathrm{~V} / \mu \mathrm{m}$, while material $\mathbf{P}_{10}(\mathbf{1} / \mathbf{3})$ had a $s_{\max }=2 \%$ at $E_{b}=30$ $\mathrm{V} / \mu \mathrm{m}$.

The actuation strains at $30 \mathrm{~V} / \mu \mathrm{m}$ (see Table 5) for materials $\mathbf{P}_{\mathbf{x}} \mathbf{( 1 / 3 )}$ are only slightly higher than for materials $\mathbf{P}_{\mathbf{x}}(\mathbf{1} / \mathbf{2})$, despite that $P_{x}(\mathbf{1 / 3})$ are softer as compared to $P_{x}(\mathbf{1 / 2})$. These small differences are due to the higher $\varepsilon^{\prime}$ observed for materials $P_{\mathbf{x}}(\mathbf{1} / \mathbf{2})$ which compensate the increase in the elastic moduli. Since the stress-strain curves of materials $\mathbf{P}_{\mathbf{6 2}}(\mathbf{1 / 3})$ and $\mathbf{P}_{\mathbf{1 0}}(\mathbf{1} / \mathbf{3})$ were superimposable, a direct comparison of the effect of $\varepsilon^{\prime}$ on the actuation is possible. Material $\mathbf{P}_{\mathbf{6 2}}(\mathbf{1 / 3})$ has an $\varepsilon^{\prime}=$ 4 and an actuation strain of $3.2 \%$ at $34 \mathrm{~V} / \mu \mathrm{m}$, while material $\mathbf{P}_{10}(\mathbf{1} / \mathbf{3})$ has an $\varepsilon^{\prime}=2.9$ and an actuation strain of $1.6 \%$ at the same electric field. The better performance in the actuation strain for material $\mathbf{P}_{\mathbf{6 2}}(\mathbf{1 / 3})$ as compared to $\mathbf{P}_{\mathbf{1 0}}(\mathbf{1 / 3})$ is due to the higher $\varepsilon^{\prime}$. The actuation curves for materials $P_{\mathbf{6 2}}(\mathbf{1 / 2})$ and $\mathbf{P}_{\mathbf{0}}(\mathbf{1 / 3})$ are also superimposable despite that material $\mathbf{P}_{\mathbf{6 2}}(\mathbf{1 / 2})$ has a higher elastic moduli as compared to $P_{0}(\mathbf{1} / \mathbf{3})(0.8 \mathrm{MPa}$ and 0.52 $\mathrm{MPa}$, respectively). Also here, the improved actuation performance of $\mathbf{P}_{\mathbf{6 2}}(\mathbf{1 / 2})$ is due to the higher $\varepsilon^{\prime}$.
When preformed polar particles were blended in the PDMS matrix as was the case for material $\mathbf{P}_{62}{ }^{\text {part }}(\mathbf{1 / 2})$, the actuation strain at $30 \mathrm{~V} / \mu \mathrm{m}$ increased as compared to the interconnected network with similar composition $\mathbf{P}_{\mathbf{6 2}}(\mathbf{1} \mathbf{2})$. However, the electrical breakdown in the actuator occurred at lower voltage and the maximum actuation strain decreased.

It is worth mentioning that the breakdown in actuator for the networks with high content of polar components $\mathbf{P}_{\mathbf{6 2}} \mathbf{( 1 / 2 )}$ and $P_{\mathbf{1 0}}(\mathbf{1 / 2})$ is higher than for the PDMS reference.

The breakdown fields of the materials $\mathbf{P}_{\mathbf{x}}(\mathbf{y} / \mathbf{z})$ were lower as compared to the PDMS reference (Table 5). The $E_{\mathrm{EB}}$ of the PDMS was $128 \mathrm{~V} / \mu \mathrm{m}$, while the breakdown of materials $\mathbf{P}_{\mathbf{x}}(\mathbf{y} / \mathbf{z})$ occurred at approximately half of the field supported by pristine PDMS, e.g. all materials $\mathbf{P}_{\mathbf{x}}(\mathbf{y} / \mathbf{z})$ have $E_{E B}$ between 54 and $71 \mathrm{~V} / \mu \mathrm{m}$, except for materials obtained with $\mathbf{P}_{\mathbf{8 9}}$. These are high values, comparable with those recently reported for polysiloxanes modified with 1-ehtynyl4-nitrobenzene [30].

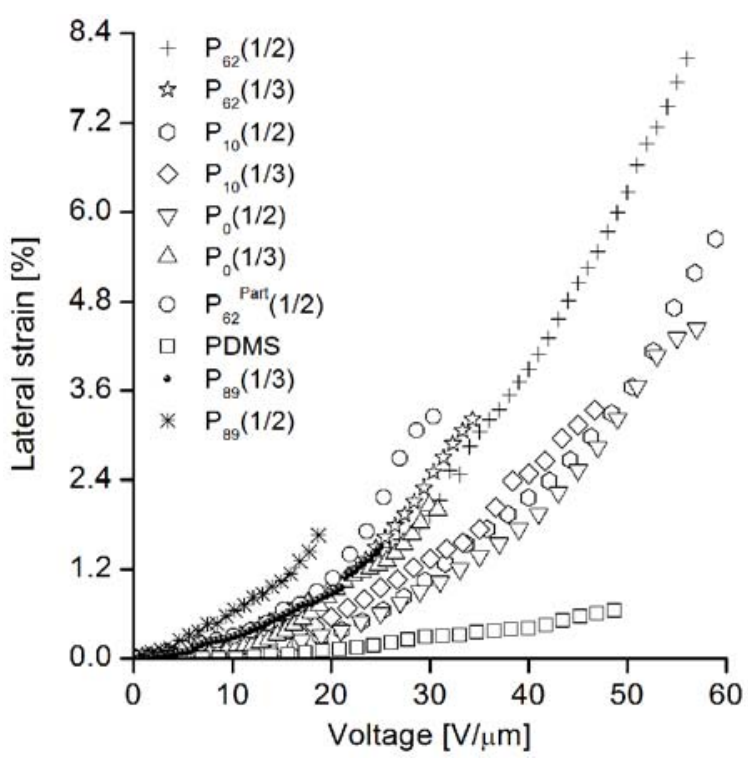

Fig. 5 Lateral actuation strain of circular actuators of materials $\mathbf{P}_{\mathbf{x}}(\mathbf{y} / \mathbf{z}), \mathbf{P}_{\mathbf{6 2}}{ }^{\text {Part }}(\mathbf{1} / \mathbf{2})$ and PDMS reference as a function of applied electric field. 
A direct comparison between the performance of materials $\mathbf{P}_{\mathbf{x}}(\mathbf{y} / \mathbf{z})$ and that of other similar materials reported in the literature is difficult to be done since detailed description of the material properties is often missing. Additional complications arise due to different setups used for testing the materials, different crosslinking methods and different prestrains used in the actuators. Given the structure similarity of the polar component $\mathbf{P}_{\mathbf{x}}$ used in this work with the previously reported one [31], a comparison can be done regarding the incorporation of the polar phase into the matrix (i.e. interconnected networks versus blending). It seems that by chemically bonding the polar component to the nonpolar PDMS, materials with increased electromechanical stability are formed, in spite of the observed microphase separation. The high values obtained for the electrical breakdown recommend these materials as dielectrics in transducers applications.

The actuation strain in the thickness direction can be calculated according to equation (1) [47], where $E$ is the electric field, $\varepsilon_{0}$ $=8.854 \cdot 10^{-12} \mathrm{~F} \mathrm{~m}^{-1}$ is the vacuum permittivity, $\varepsilon^{\prime}$ and $Y$ are permittivity and modulus of the sample:

$s_{z}=-\frac{\varepsilon_{0} \varepsilon^{\prime}}{Y} E^{2}$

Assuming incompressibility, it is also possible to estimate the lateral actuation strain $\left(s_{x}\right)$ according to equation (2):

$s_{x}=\left(1+s_{z}\right)^{-0.5}-1$

The calculated and experimental results are shown in Fig. 10S, for a lateral actuation strain at $30 \mathrm{~V} / \mu \mathrm{m}$ and maximum actuation, respectively. A good correlation was obtained for both plots. With rising content of polar groups the maximum actuation strain elevates, which reflects the increase of the dielectric $\varepsilon^{\prime}$. The exception is sample $\mathbf{P}_{\mathbf{6 2}}(\mathbf{1 / 3})$, for which both the calculated and the experimental values of $s_{x}$ are lower than expected, due to the lower breakdown in actuator.

Dielectric elastomers can also be used as generators to convert mechanical energy into electrical energy (operation known as energy harvesting or scavenging). For estimative calculation of the energy that could be generated, various models were proposed and different mechanisms of failure are considered [48]. The evaluation of the energy conversion can be done using equation (3) and assuming a series of approximations (see for example [29, 49]). The specific energy per unit volume is a function of dielectric permittivity, breakdown field and maximum strain at break $\left(s_{\max }\right)$.

$$
\frac{\Delta W_{\max }}{V}=\frac{1}{2} \varepsilon \varepsilon_{0} E_{\max }^{2}\left(1-\frac{1}{\left(s_{\max }+1\right)^{2}}\right)
$$

Assuming $E_{\max }$ is the dielectric strength $\left(E_{E B}\right)$, the maximum energy difference calculated represents the theoretical maximum value that can be achieved. It is obvious that in practice lower voltages have to be applied, to avoid material failure. A more reasonable value for the $E_{\max }$ could be the $E_{b}$ in actuator. Maximum strain at break is also a limit that involves material rupture and therefore calculations for an arbitrary $100 \%$ strain for all samples were also included, to allow comparison. The energy difference per volume unit gained during one working cycle, calculated using the $E_{b}$ in actuation or the $E_{E B}$ and either $s_{\max }$ or $s=100 \%$ are given in Table 6 . The best values for the energy were obtained for $\mathbf{P}_{\mathbf{6 2}}(\mathbf{1} / \mathbf{2})$, which has the highest $\varepsilon^{\prime}$ and high breakdown field. There is a clear increase in energy gain for the materials containing polar groups compared to the ones based on $\mathbf{P}_{\mathbf{0}}$.

Table 6: The energy difference estimated with equation (3) by using the dielectric permittivity at $10 \mathrm{kHz}$

\begin{tabular}{|c|c|c|c|}
\hline Sample & 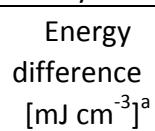 & $\begin{array}{c}\text { Energy } \\
\text { difference } \\
{\left[\mathrm{mJ} \mathrm{cm}^{-3}\right]^{\mathrm{b}}}\end{array}$ & $\begin{array}{c}\text { Maximum energy } \\
\text { difference } \\
{\left[\mathrm{mJ} \mathrm{cm}{ }^{-3}\right]^{c}}\end{array}$ \\
\hline$P_{89}(1 / 2)$ & 7.0 & 5.9 & 16.3 \\
\hline$P_{89}(1 / 3)$ & 10.5 & 8.7 & 19.5 \\
\hline$P_{62}(1 / 2)$ & 53.9 & 46.9 & 64.0 \\
\hline$P_{62}(1 / 3)$ & 18.3 & 15.4 & 53.3 \\
\hline$P_{10}(1 / 2)$ & 41.1 & 34.7 & 43.9 \\
\hline$P_{10}(1 / 3)$ & 26.2 & 21.3 & 59.9 \\
\hline$P_{0}(1 / 2)$ & 12.3 & 10.4 & 31.0 \\
\hline$P_{0}(1 / 3)$ & 9.6 & 8.0 & 38.6 \\
\hline$P_{62}{ }^{\text {part }}(1 / 2)$ & 15.3 & 12.5 & 66.0 \\
\hline${ }^{\text {soft }} P_{62}(1 / 3)$ & 24.0 & 18.9 & 66.7 \\
\hline${ }^{\text {soft }} P_{62}{ }^{\text {part }}(1 / 2)$ & 36.4 & 28.1 & 52.0 \\
\hline PDMS & 23.4 & 20.7 & 159.4 \\
\hline
\end{tabular}

a calculated with $E_{\max }=E_{b}$ in actuation and $s_{\max }$ at break

${ }^{\mathrm{b}}$ calculated with $E_{\max }=E_{b}$ in actuation and $s_{\max }=100 \%$

${ }^{c}$ calculated with $E_{\max }=E_{E B}$ and $s_{\max }$ at break

The silicone film from PolyPower ${ }^{\mathrm{TM}}$ was investigated regarding the energy scavenging [50]. The modeled and experimental energy generated per cycle by an element having a roughly estimated volume of ca. $3 \mathrm{~cm}^{3}$ at a maximum strain of $15 \%$ and $1800 \mathrm{~V}$ was ca. $94 \mathrm{~mJ}$. The calculated energy would place our materials within the range of promising materials for energy harvesting. Since this is just a rough estimation of the harvesting potential, further work is needed to measure experimentally the energy gained.

\section{Optimized materials}

As discussed above, the silicone matrix used was rather stiff (see Table 4). It is therefore possible to further optimize the mechanical properties by using a PDMS of a higher $M_{w}$ or by lowering the amount of TEOS used. Preliminary results show that much softer materials with higher strains at break can be prepared (see Supp. Inf). For example, when the amount of TEOS was reduced from ca. $50 \%$ to $20 \%$ reported to the mass of polymers (material ${ }^{\text {soft }} \mathbf{P}_{62}(\mathbf{1} / \mathbf{3})$ ), the mechanical properties were significantly improved: the strain at break was increased to $350 \%$ and the Young's modulus at $10 \%$ strain was only $0.275 \mathrm{MPa}$ (Table $1 \mathrm{~S}$ ).

When preformed polar particles were dispersed in a PDMS of a $M_{w}=400 \mathrm{kDa}$, an even softer material was obtained $\left({ }^{\text {soft }} \mathbf{P}_{62}{ }^{\text {part }}(\mathbf{1 / 2})\right)$, with elongation at break as high as $489 \%$, tensile strength of ca. 0.6 $\mathrm{MPa}$ and Young's modulus as low as $0.14 \mathrm{MPa}$ 
The electromechanical performance of these materials was improved. For example, the maximum actuation strain almost doubled for ${ }^{\text {soft }} \mathbf{P}_{\mathbf{6 2}}(\mathbf{1} / \mathbf{3})$ compared to $\mathbf{P}_{\mathbf{6 2}}(\mathbf{1 / 3})$ (6.3\% versus $\left.3.2 \%\right)$. The estimated harvesting performance of ${ }^{\text {soft }} \mathbf{P}_{\mathbf{6 2}}(\mathbf{1 / 3})$ is also better than its corresponding reference (Table 6). For the blends with polar particles, the actuation strain at $30 \mathrm{~V} / \mu \mathrm{m}$ was similar, while the maximum actuation strain was double when $400 \mathrm{kDa}$ PDMS was used as the matrix (Figure 11S).

\section{Conclusions}

Polysiloxanes $\mathbf{P}_{\mathbf{x}}$ with varying mol\% of polar cyano groups were used as high permittivity components in a high molecular weight PDMS matrix. Incorporation of a large amount of polar $\mathbf{P}_{\mathbf{x}}$ was possible by using either a three-step process that allows formation of interconnected networks or by using readily dispersible polar nanoparticles. By using these approaches the macroscopic phase separation was avoided and materials with increased permittivity and good mechanical properties formed. Additionally, the dielectric breakdown of these materials was significantly higher as compared to similar reported materials prepared by blending a polar filler into nonpolar PDMS matrix. Furthermore, materials with up to 13-times higher actuation strain as compared to the silicone matrix were obtained. The actuation strain can be easily tuned by using different amount and type of $\mathbf{P}_{\mathbf{x}}$. Theoretical calculations predict that these materials might be suitable for energy harvesting.

\section{Acknowledgements}

We gratefully acknowledge the financial support of Swiss National Science Foundation and UEFISCDI, under the Swiss-Romanian Cooperation Program, grant No. IZERZO_142215 / 1 (10 / RO$\mathrm{CH} / \mathrm{RSRP} / 01.01 .2013)$ and SNFS, grant No. 150638, COST Action MP1003, and Swiss Federal Laboratories for Materials Science and Technology (Empa, Dübendorf). One of the authors (C.-D.V.) acknowledges the financial support of a grant of the Romanian National Authority for Scientific Research, project number PN-II-IDPCCE-2011-2-0028. We also acknowledge Dr. Rentsch for his kind support with the NMR investigations.

\section{References}

1. T. Mirfakhrai, J. D. W. Madden and R.H. Baughman, Materials Today 2007, 10, 30-38.

2. Y. Bar-Cohen, Electroactive Polymer (EAP) Actuators as Artificial Muscles-Reality, Potential and Challenges, SPIE Optical Engineering Press, Bellingham, WA, 2001.

3. S. G. Wax and R. R. Sands, Proc. SPIE 1999, 3669, 2-10.

4. J. Eckerle, S. E. Stanford, J. P. Marlow, R. Schmidt, S. Oh, T. P. Low and S. V. Shastri, Proc. SPIE 2001, 4332, 269-280.

5. R. Pelrine, R. Kornbluh, Q. Pei, S. Stanford, S. Oh, J. Eckerle, R. Full, M. Rosenthal and K. Meijer, Proc. SPIE 2002, 4695, 126-137.

6. Q. Pei, M. Rosenthal, R. E. Pelrine, S. E. Stanford and R. D. Kornbluh, Proc. SPIE 2003, 5051, 281-290.

7. S. Ashley, Sci. Am. 2003, 289, $52-59$.

8. R. Kornbluh, R. Pelrine, Q. Pei, and V. Shastri, In Artificial Muscles Reality, Potential and Challenges, Y. Bar-Cohen (Ed.), SPIE Press, Bellingham, Washington, DC, 2001, ch. 16, pp. $457-503$.
9 H. Herr and R. Kornbluh, Smart Structures and Materials: Electroactive Polymer Actuators and Devices, San Diego, CA., 2004.

10. M. Palmer, M.S. Thesis, Department of Mechanical Engineering, MIT, Boston, MA., 2002.

11. L. L. Creswell, M. Rosenbloom, J. L. Cox, T. B. Ferguson, N. T. Kouchoukos, T. L. Spray, M. K. Pasque, T. B. Ferguson, T. H. Wareing and C. B. Huddleston, Ann. Thorac. Surg. 1992, 54, 11-18.

12. F. D. Pagani, K. D. Aaronson, D. B. Dyke, S. Wright, F. Swaniker, R. H. Bartlett, Ann. Thorac. Surg. 2000, 70, 19771985.

13. F. Carpi, and D. De Rossi, Proceedings of the Electroactive Polymer Actuators and Devices (EAPAD), San Diego, CA, p. 65240D, 2007.

14. C. Menon, F. Carpi and D. De Rossi Acta Astronaut. 2009 4329, 825-833.

15. R. D. Kornbluh, D. S. Flamm, H. Prahlad, K. M. Nashold, S. Chhokar, R. Pelrine, D. L. Huestis, J. Simons, T. Cooper and D. G. Watters Proc. SPIE 2003, 5051, 143-158.

16 G. J. Lin, X. B. Zhang and D. C. Song, Adv. Mater. Res. 2011, 328-330, 1491-1494.

17. R. Yang, Y. Qin, C. Li, G. Zhu and Z. L. Wang, Nano Lett. 2009, 9, 1201-1205.

18. P. Harsha, R. Kornbluh, R Pelrine, S. Stanford, J. Eckerle and S. Oh Proc of Int. Conf. on Smart Materials Structures and Systems, Bangalore, 2005.

19. S. Chiba, M. Waki, R. Kormbluh and R. Pelrine Proc. SPIE 2008, 6927, 1-9.

20. R. Vertechy, M. Fontana, G. P. Rosati Papini, and M. Bergamasco, Proc. SPIE 2013, 8687, doi:10.1117/12.2012016.

21. J. Maas and C. Graf, , Smart Mater. Struct. 2012, 21064006.

22. B. Kussmaul, S. Risse, M. Wegener, M. Bluemke, J. Krause, J. Wagner, T. Feller, K. Clauberg, J. Hitzbleck, R. Gerhard, H. Krueger, Proc. SPIE 2013, 8687, Electroactive Polymer Actuators and Devices (EAPAD), 86872S, doi:10.1117/12.2009102.

23. R. Kornbluh and R. Pelrine, High-performance acrylic and silicone elastomers, in:, Dielectric elestomers as electromechanical transducers, F. Carpi, D. De Rossi, R. Kornbluh, R. Pelrine, P. Sommer-Larsen (Eds.), Elsevier, Pisa, 2007

24. M. G. Voronkov, V. P. Mileshkevich and Y. A. Yuzhelevskii, The Siloxane Bond: Physical Properties and Chemical Transformations, Consultants Bureau, New York, 1978.

25. R. G. Jones, W. Ando, and J. Chojnowski (Eds.) SiliconContaining Polymers: The Science and Technology of Their Synthesis and Applications, Kluwer Academic Publishers, Dordrecht, The Netherlands, 2000.

26 W. Noll, Chemistry and Technology of Silicones, Academic Press, New York and London, 1968.

27 A. C. M. Kuo, Poly(dimethylsiloxane), in Polymer Data Handbook. Oxford University Press, 1999, pp. 411-435.

28 A. P. Mulgaonkar, R. Kornbluh and H. Herr, Biomedical, Haptic and Micro-Scale Applications, in: Dielectric elestomers as electromechanical transducers, F. Carpi, D. De 
Rossi, R. Kornbluh, R. Pelrine, P. Sommer-Larsen (Eds.), Elsevier, Pisa, 2007.

29 M. Molberg, D. Crespy, P. Rupper, F. Nüesch,J. A. E. Månson, C. Löwe and D. M. Opris, Adv. Funct. Mater. 2010, 20, 32803291.

30 F. Bahrt Madsen, L. Yu, A. E. Daugaard, S. Hvilsted, A. Ladegaard Skov, Polymer 2014, 55, 6212-6219.

31 S. Risse, B. Kussmaul, H. Krüger and G. Kofod, Adv. Funct. Mater. 2012, 22, 3958-3962.

32 F. B. Madsen, I. Dimitrov, A. Daugaard, S. Hvilsted and A. L. Skov, Polym. Chem. 2013, 4, 1700-1707.

33 S. Dünki, Y. S. Ko, F. A. Nüesch, D. M. Opris, Adv. Funct. Mater. 2015, 25, 2467; S. J. Dünki, M. Tress, F. Kremer, S. Y. Ko, F. A. Nüesch, C.-D. Varganici, C. Racles, D. M. Opris; RSC Adv. 2015, 5, 50054.

34. C. Racles, M. Cazacu, B. Fischer and D. M. Opris, Smart. Mater. Struct. 2013, 22, 104004.

35. C. Racles, M. Alexandru, A. Bele, V. E. Musteata, M. Cazacu and D. M. Opris, RSC Adv. 2014, 4, 37620-37628.

36 C. Racles, Soft Materials 2010, 8, 1-11.

37 M. Kollosche and G. Kofod, Appl. Phys. Lett. 2010, 96, 071904.

38 A. L. Smith, Analysis of silicones, vol 41 in Chemical Analysis, A series of monographs on analytical chemistry and its aplications, A. L. Smith (Ed.), Wiley, USA, 1974.

39 I. Yilgor and J. E. McGrath, Adv. Polym. Sci. 1988, 86, 1-86.

40 C. Racles, A. Ioanid, A. Tóth, M. Cazacu and V. Cozan, Polymer 2004, 45, 4275-4283.

41 C. D. Varganici, O. Ursache, C. Gaina, V. Gaina and B.C. Simionescu, J. Therm. Anal. Calorim. 2013, 111, 1561-1570.

42 C. D. Varganici, L. Rosu, D. Rosu and B.C. Simionescu, Compos. Part B.: Eng. 2013, 50, 273-278.

43 P. R. Sundarajan, Polymer, 2002 43, 1691-1693.

44 M. Alexandru, M. Cristea, M. Cazacu, A. loanid and B.C. Simionescu, Polym. Composite. 2009, 30, 751-759.

45 F. Carpi, G. Gallone, F. Galantini and D. De Rossi, Adv. Funct. Mater. 2008, 18, 235-241.

46 A. K. Jonscher, J. Phys. D: Appl. Phys. 1999. 32, R57-R70.

47 R. E. Pelrine, R. D. Kornbluh, J. P. Joseph, Sensors and Actuators A 1998, 64, 77-85.

48. S. J. A. Koh, C. Keplinger, T. Li, S. Bauer and Z. Suo, IEEE/ASME Transactions on Mechatronics 2011, 16, 33-41.

49. S. J. A. Koh, X. Zhao and Z. Suo, Appl. Phys. Let. 2009, 94, 262902.

50. Y. H. Iskandarani, R. W. Jones and E. Villumsen, Proc. of SPIE 2009, 7287, 72871Y, doi: 10.1117/12.815267. 